\title{
Spin-glass-like ordering of the magnetic moments of interacting nanosized maghemite particles
}

\author{
Mørup, Steen; Bødker, Franz; Hendriksen, Peter Vang; Linderoth, Søren
}

Published in:

Physical Review B

Link to article, DOI:

10.1103/PhysRevB.52.287

Publication date:

1995

Document Version

Publisher's PDF, also known as Version of record

Link back to DTU Orbit

Citation (APA):

Mørup, S., Bødker, F., Hendriksen, P. V., \& Linderoth, S. (1995). Spin-glass-like ordering of the magnetic moments of interacting nanosized maghemite particles. Physical Review B, 52(1), 287-294.

https://doi.org/10.1103/PhysRevB.52.287

\section{General rights}

Copyright and moral rights for the publications made accessible in the public portal are retained by the authors and/or other copyright owners and it is a condition of accessing publications that users recognise and abide by the legal requirements associated with these rights.

- Users may download and print one copy of any publication from the public portal for the purpose of private study or research.

- You may not further distribute the material or use it for any profit-making activity or commercial gain

- You may freely distribute the URL identifying the publication in the public portal 


\title{
Spin-glass-like ordering of the magnetic moments of interacting nanosized maghemite particles
}

\author{
S. Mørup, F. Bødker, P. V. Hendriksen,* and S. Linderoth* \\ Physics Department, Building 307, Technical University of Denmark, DK-2800 Lyngby, Denmark
}

(Received 19 December 1994)

\begin{abstract}
Samples of interacting nanosized maghemite particles have been studied by Mössbauer spectroscopy and magnetization measurements. The apparent blocking temperatures obtained from Mössbauer spectroscopy and zero-field-cooled magnetization curves are nearly identical, but the values obtained from measurements of the decay of remanence are much lower. These results are not in accordance with the Néel model for superparamagnetic relaxation, but can be explained by the formation of an ordered spinglass-like state at low temperatures. At a critical temperature a transition to a superparamagnetic state takes place. By varying the average interparticle distance it is possible to change the ordering temperature.
\end{abstract}

\section{INTRODUCTION}

Nanometer-sized particles of magnetic materials are superparamagnetic at finite temperatures. For noninteracting particles with uniaxial magnetic anisotropy, the superparamagnetic relaxation can be described by the Néel expression ${ }^{1}$

$$
\tau=\tau_{0} \exp \left\{\frac{K V}{k T}\right\},
$$

where $k$ is Boltzmann's constant, $T$ is the temperature, $K$ is the magnetic anisotropy energy constant, and $V$ is the particle volume. $\tau_{0}$ is typically of the order of $10^{-10}-10^{-12}$ s. $^{2-4}$ Superparamagnetic relaxation can conveniently be studied by using Mössbauer spectroscopy and magnetization measurements. When using Mössbauer spectroscopy, it is found that the superparamagnetic relaxation results in a broadening of the absorption lines for relaxation times of the order of $10^{-8} \mathrm{~s}$, and for $\tau \lesssim 10^{-9} \mathrm{~s}$, the magnetic hyperfine splitting disappears. Because of the particle size distribution in the samples, there will normally be a broad distribution of relaxation times [cf. Eq. (1)]. The blocking temperature $T_{B}^{M}$ is often defined as the temperature at which $50 \%$ of the spectral area is magnetically split. This corresponds to an average relaxation time of about $5 \times 10^{-9} \mathrm{~s}$.

When studying superparamagnetic relaxation by use of magnetization measurements, a common technique is to measure the zero-field-cooled (ZFC) magnetization, i.e., the magnetization in a small applied field as a function of increasing temperature after the sample has been cooled in a zero field. The ZFC magnetization will exhibit a maximum at the blocking temperature $T_{B}^{m}$ at which the relaxation time equals the time scale of the magnetization measurements (10-100 s). A distribution in particle size will result in a shift of the maximum to a temperature $T_{\max }=\beta T_{B}^{m}$ where $T_{B}^{m}$ is the blocking temperature for particles with an average volume. ${ }^{5,6}$ Typically, $\beta$ is of the order of $1.5-2.0 .^{6}$

When superparamagnetic particles are brought close together, the magnetic interaction (dipole and/or ex- change interaction) will affect the superparamagnetic relaxation. Interaction effects have been studied in several systems based on antiferromagnetic, ${ }^{7,8}$ ferrimagnetic, ${ }^{5,8-16}$ and ferromagnetic ${ }^{17-20}$ particles in the form of powders, ${ }^{7-9}$ solidified suspensions, ${ }^{5,10-16}$ or granular solids. ${ }^{17-20}$ Several studies have revealed an apparent increase in the blocking temperatures $T_{B}^{M}$ and $T_{B}^{m}$ when the average interparticle distances are decreased. This suggests that the relaxation time increases when the interactions are enhanced. This interpretation is in accordance with theoretical models by Shtrikman and Wohlfarth ${ }^{21}$ and by Dormann, Bessasis, and Fiorani. ${ }^{19}$ However, recent Mössbauer studies ${ }^{11,12}$ of samples of weakly interacting maghemite particles have shown that the blocking temperature $T_{B}^{M}$ decreases with increasing strength of the interaction. A model for the influence of interactions on the superparamagnetic relaxation time was derived which could explain the Mössbauer results. ${ }^{12}$ The same samples showed, however, an increase in the temperature of the maximum in the ZFC magnetization with increasing strength of the interactions, i.e., an apparent increase in the relaxation time. ${ }^{11}$ The values of $T_{\max }$ were much lower than the values of $T_{B}^{M}$. It has been suggested that the maximum in the ZFC magnetization for the concentrated samples should be explained by an ordering temperature instead of superparamagnetic blocking. ${ }^{12}$

Calculations on three-dimensional cubic arrays of dipoles, which interact only via dipolar interactions, have shown that magnetically ordered states exist below a critical temperature. ${ }^{22-24}$ When the dipoles form a simple cubic lattice, the ground state is antiferromagnetic, and for the fcc and bcc lattices, it is ferromagnetic. When the dipoles form chain-like structures, the ordering will also be ferromagnetic. ${ }^{25-27}$ In samples with a random distribution of magnetic dipoles, the ordered state will resemble a spin glass. ${ }^{7,9,13,23,28}$ For magnetic moments of the order of a few Bohr magnetons, the ordering temperatures will be in the millikelvin range, but for particles with magnetic moments of several thousand Bohr magnetons, the ordering temperature can be much higher. If the particles are in close contact, the exchange interaction between surface atoms belonging to neighboring par- 
ticles may be significant and this can further increase the ordering temperature.

The onset of this type of ordering in materials containing nanometer-sized magnetic particles may be difficult to distinguish from superparamagnetic blocking of noninteracting or weakly interacting particles. In Mössbauer spectroscopy studies, one will find the disappearance of the magnetic hyperfine splitting both at the blocking temperature of superparamagnetic particles and at the ordering temperature of strongly interacting particles if $\tau \lesssim 10^{-9} \mathrm{~s}$ above the ordering temperature. ZFC magnetization measurements will exhibit a maximum close to the blocking temperature of superparamagnetic particles, and for strongly interacting particles a maximum will be found at the ordering temperature if $\tau \lesssim 10 \mathrm{~s}$ above this temperature.

Because the time scale of Mössbauer spectroscopy is much shorter than that of magnetization measurements, the blocking temperature $T_{B}^{M}$ of noninteracting or weakly interacting particles is much higher than $T_{B}^{m}$. When the strength of the interaction is intermediate, one may observe superparamagnetic blocking in Mössbauer experiments while the maximum in the ZFC magnetization curve may be due to ordering. In the case of strong interactions, the critical temperatures found in both Mössbauer and magnetization measurements may be due to ordering. The behavior of magnetic nanocomposites as a function of the strength of the interactions has been discussed in more detail in a recent publication. ${ }^{29}$

In this paper the effects of interactions between nanometer-sized maghemite $\left(\gamma-\mathrm{Fe}_{2} \mathrm{O}_{3}\right)$ particles are examined by combining Mössbauer spectroscopy and magnetization measurements. Studies were made of coated particles both in suspension and in dry powder form and of uncoated dried particles. The effect of pressing the dry samples was also investigated. Both the drying and the pressing are expected to decrease the average interparticle distance and thus result in stronger particle interactions. Some preliminary results have been published earlier. ${ }^{30}$ The same maghemite particles have also been used for studies of spin canting. ${ }^{31}$

\section{EXPERIMENTAL DETAILS}

The initial sample, consisting of magnetite $\left(\mathrm{Fe}_{3} \mathrm{O}_{4}\right)$ particles, was prepared by coprecipitation of $\mathrm{Fe}(\mathrm{II})$ and $\mathrm{Fe}$ (III) in an aqueous solution. Half of the sample was coated with oleic acid, and then both samples were washed with water and dried. Before the drying some of the coated particles were separated and suspended in vacuum oil, producing a ferrofluid. Upon exposure to air, both the particles in the ferrofluid and the dried particles oxidized to maghemite. Some of the powder of both the coated and uncoated particles was uniaxialy compressed at room temperature at pressures in the range 120-1280 MPa.

The Mössbauer spectra were obtained using constantacceleration spectrometers with sources of ${ }^{57} \mathrm{Co}$ in rhodium. The spectrometers were calibrated with a $12.5-\mu \mathrm{m}$ thick $\alpha-\mathrm{Fe}$ foil at room temperature.

Magnetization measurements were carried out using a standard vibrating-sample magnetometer. The zerofield-cooled (ZFC) magnetization was obtained by cooling to about $4 \mathrm{~K}$ in zero magnetic field and then applying a small magnetic field, and measuring the magnetization $100 \mathrm{~s}$ later. The temperature was then increased in steps and the procedure repeated. For the measurement of the temperature decay of isothermal remanent magnetization (IRM), the sample was cooled to $4 \mathrm{~K}$ in zero field. A saturation field was applied-1 $\mathrm{T}$ is normally large enough - and the saturation magnetization $M_{s}(T)$ measured. The field was then removed, and $100 \mathrm{~s}$ later the remanent magnetization $M_{r}(T)$ was measured. The sample was warmed up in small steps and the procedure repeated at each temperature.

Electron micrographs were obtained using a Philips EM 301 and a Philips EM 430 microscope.

Energy-dispersive $\mathrm{x}$-ray-diffraction spectra were obtained by measuring the energy spectrum of scattered white $x$-ray radiation. From the line broadening of the diffraction peaks, an estimate of the mean crystallite size could be deduced. ${ }^{32}$

\section{RESULTS}

The particle size distribution was estimated from the room-temperature magnetization curve by using the method developed by Chantrell, Popplewell, and Charles, ${ }^{33}$ assuming a log-normal distribution of the particle diameters $d$ :

$$
f(y)=\frac{1}{(2 \pi)^{1 / 2} \sigma_{y} y} \exp \left[-\frac{(\ln y)^{2}}{2 \sigma_{y}^{2}}\right],
$$

where $y=d / d_{m}$ and $d_{m}$ is the median diameter.

The data and analysis are given in Ref. 31 . The median size $d_{m}$ was found to be $7.5 \mathrm{~nm}$ with a standard deviation $\sigma_{d}=0.43$.

The electron micrographs showed that the particles were fairly spherical. The particle size estimated from the micrographs and from the energy-dispersive $x$-raydiffraction spectra was within the experimental uncertainty in accordance with the results obtained from magnetization measurements. The coated and uncoated maghemite particles were found to have the same average diameter within experimental error.

Room-temperature Mössbauer spectra of the coated particles, obtained in various applied magnetic fields up to $0.6 \mathrm{~T}$, are shown in Fig. 1. At $B=0.01 \mathrm{~T}$, the spectrum consists essentially of a quadrupole doublet with broad lines. With increasing applied fields, a substantial magnetic hyperfine splitting is induced. Such a field dependence shows unambiguously that the particles are superparamagnetic at room temperature. ${ }^{26,34}$ From the field dependence of the magnetic hyperfine splitting, an average magnetic moment of $1.06 \times 10^{-19} \mathrm{~J} \mathrm{~T}^{-1}$ was estimated using a procedure described elsewhere. ${ }^{26,34}$ Assuming that the saturation magnetization is the same as for bulk maghemite and that the particles are spherical, this corresponds to an average particle diameter of 7.5 $\mathrm{nm}$ in agreement with the results obtained by the other techniques. 


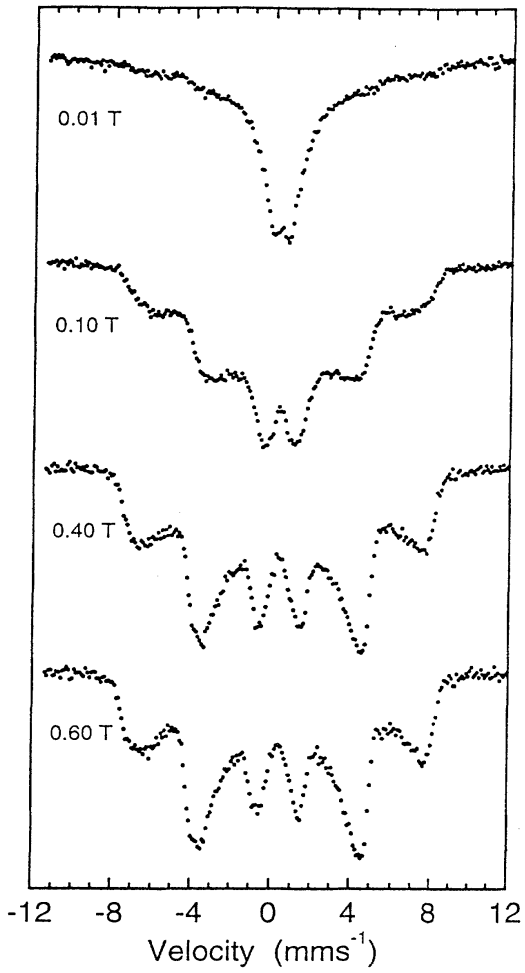

FIG. 1. Mössbauer spectra of the coated maghemite particles obtained at $295 \mathrm{~K}$ with the indicated values of magnetic fields applied perpendicular to the $\gamma$-ray direction.
Figure 2 shows Mössbauer spectra of the suspended maghemite particles in a range of temperatures well below the melting point of the carrier liquid. The magnetically split spectrum at $13 \mathrm{~K}$ is typical for maghemite particles, with a hyperfine field of about $51 \mathrm{~T}$ and a slight asymmetry in both the linewidth and intensity due to a small difference in the parameters of the two sites in the spinel structure. The observed hyperfine field is lower than for bulk maghemite (about $52.6 \mathrm{~T}$ at $4 \mathrm{~K}$ ), but for nanoparticles ${ }^{35-37}$ and thin films ${ }^{38}$ a reduced value is usually found. With increasing temperatures $(80,130$, and $176 \mathrm{~K}$ ), there is a gradual collapse of the magnetically split component to a superparamagnetic doublet. There is also an increasing degree of the asymmetric broadening of the lines of the sextet with increasing temperature.

Mössbauer spectra of the dry powders of coated and uncoated maghemite particles are shown in Fig. 3. There is relatively little difference between the spectra of the coated particles in suspended and dry form, except for a slightly slower collapse of the sextet with increasing temperature for the dry sample. A much more significant difference is observed when comparing the behavior of uncoated and coated particles. In the case of uncoated particles, no superparamagnetic doublet is present in the spectra at 80 and $130 \mathrm{~K}$ and a significant collapse of the sextet is first observed in the spectra at $234 \mathrm{~K}$. The spectra of the coated particles show a small contribution from a doublet already at $80 \mathrm{~K}$, and at $130 \mathrm{~K}$ the relative area of this contribution has increased considerably.
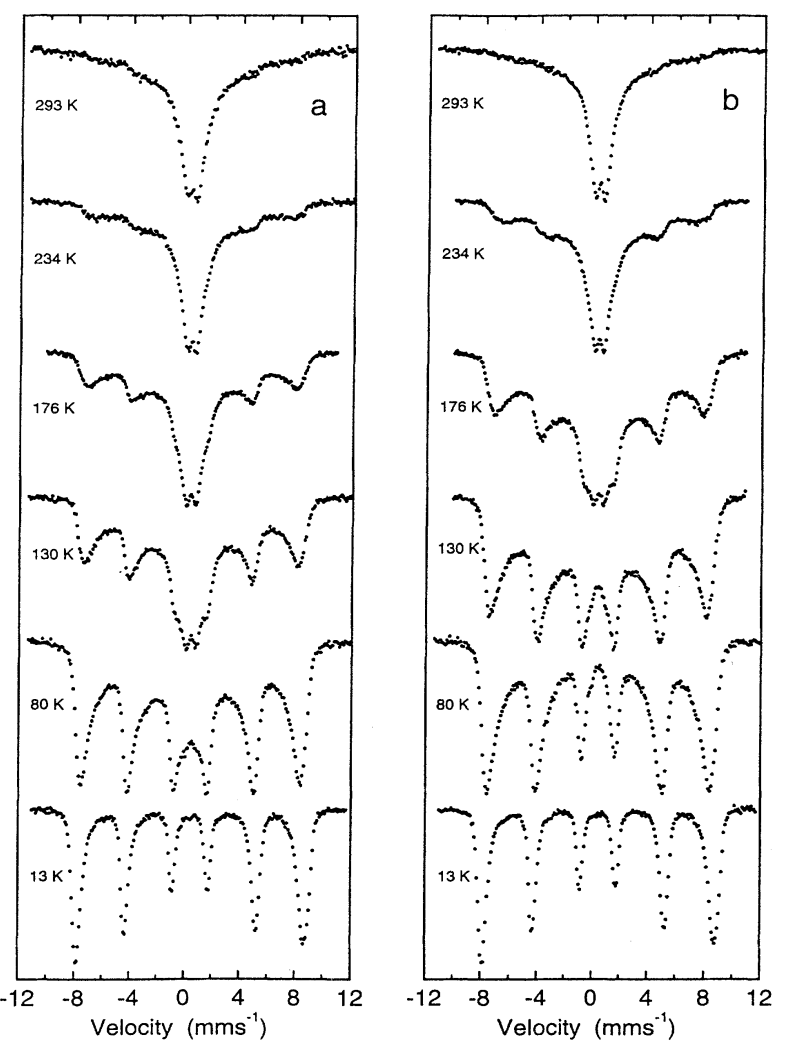

FIG. 3. Mössbauer spectra of (a) coated and (b) uncoated maghemite particles obtained at the indicated temperatures. 

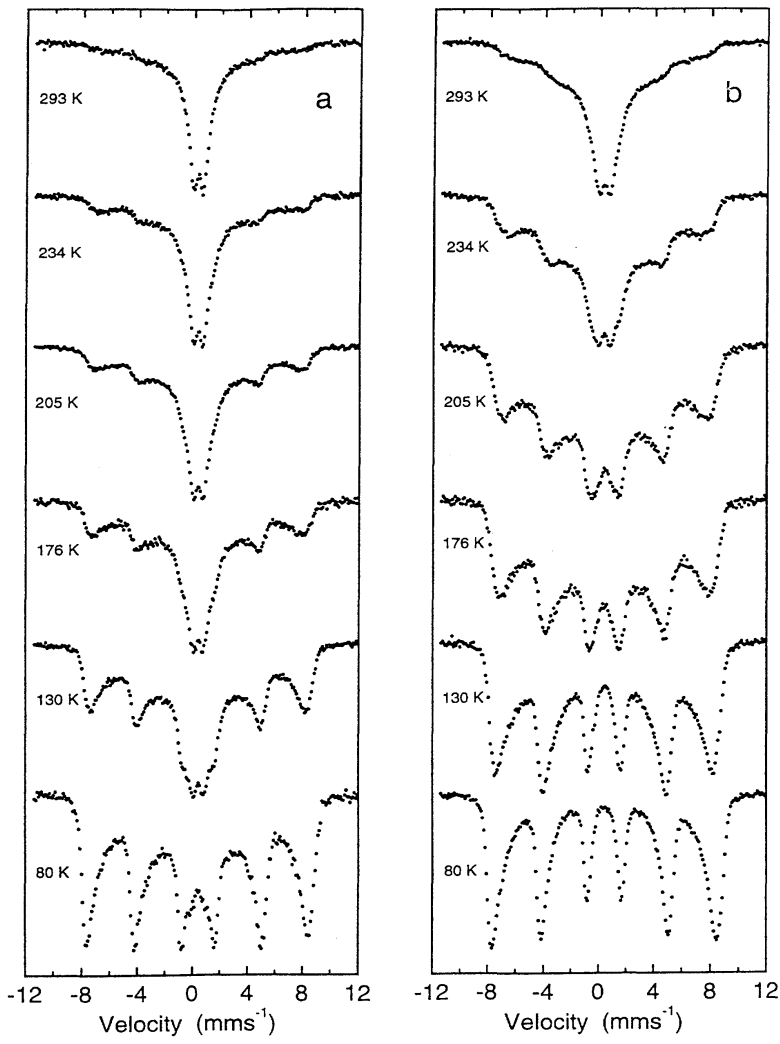

FIG. 4. Mössbaeur spectra of (a) coated and (b) uncoated maghemite particles after uniaxial pressing at 640 and 1280 $\mathrm{MPa}$, respectively. The spectra were obtained at the indicated temperatures.

Figure 4 shows Mössbauer spectra of the coated and uncoated particles after uniaxial pressing at 640 and 1280 $\mathrm{MPa}$, respectively. For the coated particles, the pressing has no visible effect on the spectra, but the effect on the uncoated sample is quite significant. Spectra with asymmetrically broadened lines, but without a visible doublet, prevail up to about $234 \mathrm{~K}$, after which there is a relatively sudden collapse of the magnetically split component. Pressing the uncoated sample with a lower pressure of $120 \mathrm{MPa}$ yields spectra (not shown) which display a behavior that is intermediate between those of the unpressed sample and the sample pressed at $1280 \mathrm{MPa}$.

The blocking temperatures were estimated by fitting the spectra with sextets and doublets. The results are given in Table I. Because the lines are broad and asymmetric, the spectra were difficult to fit with a limited number of Lorentzian lines, and therefore the uncertainties on the blocking temperatures are of the order of 30 $K$. However, the uncertainty in the difference in blocking temperatures for the samples is considerably smaller, about $5-10 \mathrm{~K}$.

The ZFC magnetization of all the samples was measured with an applied field $B_{\text {ext }}=2.2 \mathrm{mT}$ after cooling in zero field. The ZFC magnetization curves are plotted in Fig. 5. All curves have a maximum typical both for spin glasses and single-domain particles. The shift of the max-
TABLE I. Apparent blocking temperatures $T_{B}^{M}$ obtained from Mössbauer spectroscopy and the temperature of the peak in the ZFC magnetization curves, $T_{\max }$, for 7.5-nm maghemite particles in samples with different packing fractions. The ZFC magnetization curves were measured in an applied magnetic field of $2.2 \mathrm{mT}$. The uncertainty in the absolute values of $T_{B}^{M}$ is about $30 \mathrm{~K}$ and about $10 \mathrm{~K}$ for $T_{\max }$.

\begin{tabular}{llcc}
\hline \hline \multicolumn{1}{c}{ Sample } & $\begin{array}{c}T_{B}^{M} \\
(\mathbf{K})\end{array}$ & $\begin{array}{c}T_{\max } \\
(\mathbf{K})\end{array}$ & $T_{B}^{M} / T_{\max }$ \\
\hline Coated suspended & 155 & 110 & 1.4 \\
Coated dried & 170 & 148 & 1.2 \\
$\begin{array}{l}\text { Coated pressed } \\
\text { 640 MPa }\end{array}$ & 170 & 142 & 1.2 \\
$\begin{array}{l}\text { Uncoated dried } \\
\text { Uncoated pressed }\end{array}$ & 210 & 185 & 1.1 \\
$\begin{array}{l}120 \mathrm{MPa} \\
\text { Uncoated pressed }\end{array}$ & 230 & & \\
\begin{tabular}{l} 
1280 MPa \\
\hline
\end{tabular} & 265 & 210 & 1.3 \\
\hline
\end{tabular}
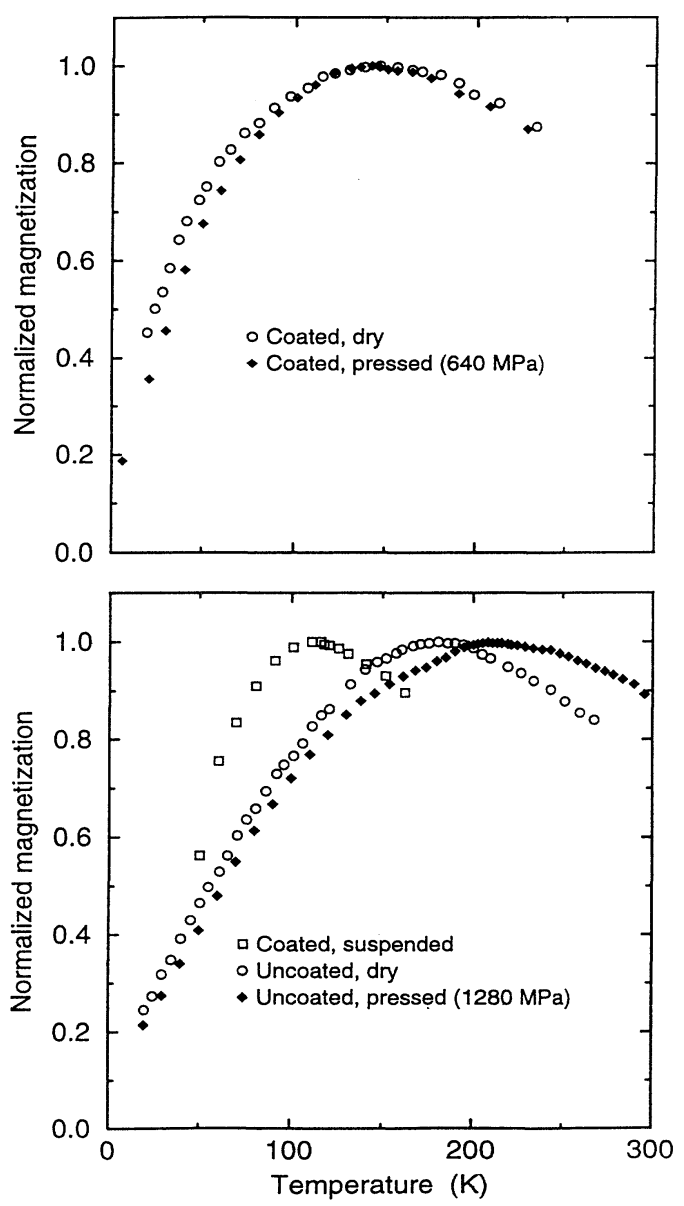

FIG. 5. ZFC magnetization curves (obtained with $B_{\text {ext }}=2.2$ $\mathrm{mT}$ ) for various samples of $7.5-\mathrm{nm}$ maghemite particles. The curves are normalized to unity at the peak value. 


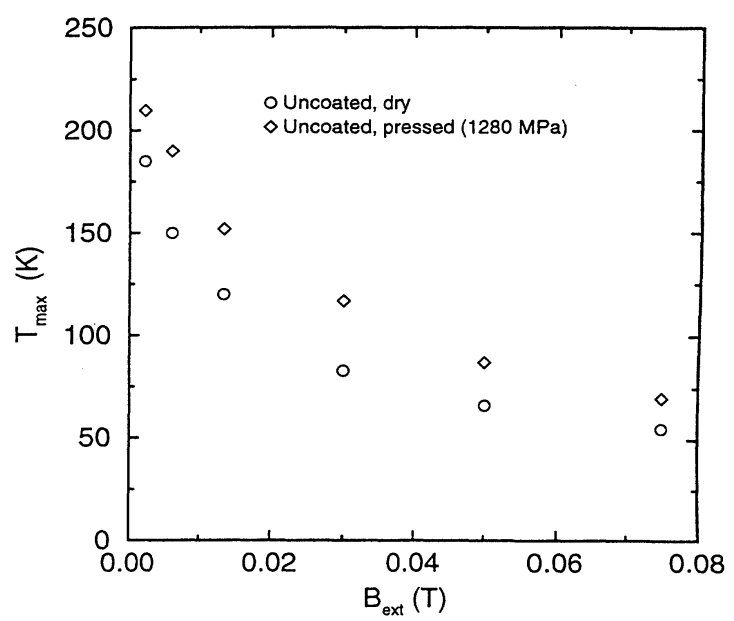

FIG. 6. Value of $T_{\max }$ as a function of $B_{\text {ext }}$ for the uncoated and uncoated pressed (1280 $\mathrm{MPa})$ samples.

imum toward higher temperatures when the interparticle distance decreases shows the same general tendency as found for the blocking temperatures obtained from the Mössbauer spectra. In addition to the shift, there is also a tendency toward increased broadening of the peak. The peak temperature $T_{\max }$ is $110 \mathrm{~K}$ for the ferrofluid and increases up to $210 \mathrm{~K}$ for the uncoated particles after pressing at $1280 \mathrm{MPa}$. The values of $T_{\max }$ are given in Table I. Earlier studies of ZFC magnetization of small particles have shown that the value of $T_{\max }$ depends on $B_{\text {ext }} \cdot{ }^{3,13,15}$ The zero-field value $T_{\max }(0)$, obtained by extrapolating to $B_{\text {ext }}=0$, should be used as a basis for a discussion of the relaxation behavior. Figure 6 shows the measured values of $T_{\max }$ as a function of $B_{\text {ext }}$ for the uncoated and uncoated pressed $(1280 \mathrm{MPa})$ samples. It is seen that $T_{\max }$ increases with decreasing values of $B_{\text {ext }}$. Extrapolation to $B_{\text {ext }}=0$ yielded values of $T_{\max }(0)$ which are $10-15 \%$ larger than the values of $T_{\max }$ obtained at $2.2 \mathrm{mT}$. Thus we can conclude that the values of $T_{B}^{M}$ and $T_{\text {max }}(0)$ are nearly identical for the powder samples.

The decay-of-remanence curves were measured for several samples with different packing fractions. Figure 7 shows the reduced remanence data as a function of temperature for the coated dry particles before and after pressing (640 $\mathrm{MPa})$ and for the uncoated particles when dried and after pressing at 240 and $1280 \mathrm{MPa}$. We have analyzed the results using the standard relation for the temperature variation of the reduced remanence (normalized to the measured saturation magnetization) given by $^{5,39}$

$$
\frac{M_{r}(T)}{M_{s}(T)}=\frac{M_{r}(0)}{M_{s}(0)} \int_{T / T_{B}^{r}}^{\infty} f(y) d y,
$$

where $T_{B}^{r}$ is the median blocking temperature, $y=T_{B} / T_{B}^{r}$ is the reduced blocking temperature, and $M_{r}(0) / M_{s}(0)$ is the reduced remanence at $0 \mathrm{~K}$. $M_{r}(0) / M_{s}(0)$ should be 0.5 for uniaxial particles with a random orientation of the easy axes. The distribution
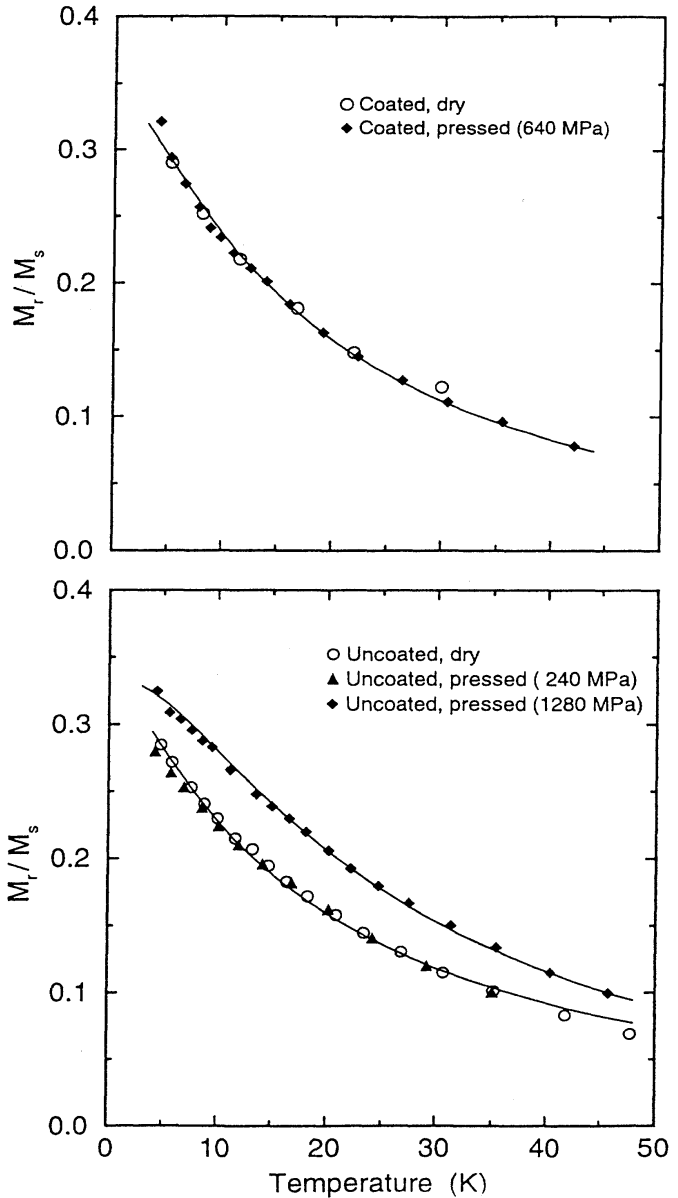

FIG. 7. Variation of the reduced isothermal remanence with temperature for the maghemite particles when (a) coated and (b) uncoated. The solid lines represent the best fit of the data with a standard decay-of-remanence model.

$f(y)$ of reduced blocking temperatures is assumed to be a log-normal function. The best fits with Eq. (3) to the data for the coated pressed, uncoated, and uncoated pressed at $1280 \mathrm{MPa}$ are shown by the lines in Fig. 7. The free parameters are $M_{r}(0) / M_{s}(0)$, the median blocking temperature, and the standard deviation $\sigma_{r}$. The values obtained are given in Table II. A relatively good fit with a standard deviation of about $\sigma_{r}=1.15$ is obtained for all samples except the uncoated sample pressed at $1280 \mathrm{MPa}$, where a lower standard deviation of about 1.0 gives a better fit. For noninteracting particles we would expect the $f(y)$ distribution to be similar to the distribution of reduced particle volumes, assuming that the change in the anisotropy constant with size and temperature is negligible. The standard deviation $\sigma_{r}$ should then be equal to $3 \sigma_{d}=1.29$, which is slightly larger than the experimentally measured value for most of the samples. For the uncoated particles which have been pressed at $1280 \mathrm{MPa}$, the deviation is larger. The values of $T_{B}^{r}$ are almost identical for the coated and uncoated, dried samples, but after pressing the uncoated particles at 1280 $\mathrm{MPa}, T_{B}^{r}$ increases significantly. 
TABLE II. Parameters obtained from a fit of the decay of remanence data for different samples of the maghemite particles, assuming a log-normal distribution of the blocking temperatures. The uncertainties are about 0.02 for the reduced remanence $M_{r}(0) / M_{s}(0)$ at $0 \mathrm{~K}$, about 0.1 for the standard deviation $\sigma_{r}$, and $2-3 \mathrm{~K}$ for the median blocking temperature $T_{B}^{r}$.

\begin{tabular}{lccc}
\hline \multicolumn{1}{c}{ Sample } & $M_{r}(0) / M_{s}(0)$ & $\sigma_{r}$ & $\begin{array}{c}T_{B}^{r} \\
(\mathrm{~K})\end{array}$ \\
\hline Coated suspended & 0.37 & 1.15 & 17 \\
Coated dried & 0.33 & 1.15 & 19 \\
Coated pressed & 0.34 & 1.15 & 19 \\
640 MPa & & & \\
Uncoated dried & 0.32 & 1.15 & 20 \\
Uncoated pressed & 0.30 & 1.15 & 21 \\
$\begin{array}{l}\text { 240 MPa } \\
\text { Uncoated pressed }\end{array}$ & 0.33 & 1.0 & 27 \\
1280 MPa & & & \\
\hline \hline
\end{tabular}

\section{DISCUSSION}

For noninteracting superparamagnetic particles, the blocking temperatures, estimated from Mössbauer spectroscopy and magnetization measurements, differ considerably because of the different time scales of the two techniques. By use of Eq. (1), one finds that the ratio between the two blocking temperatures is given by

$$
\frac{T_{B}^{M}}{T_{B}^{m}}=\frac{\ln \left(\tau_{m} / \tau_{0}\right)}{\ln \left(\tau_{M} / \tau_{0}\right)},
$$

where $\tau_{m}$ and $\tau_{M}$ are the time scales of magnetization measurements and Mössbauer spectroscopy, respectively. The value of $\tau_{0}$ is in the range $10^{-10}-10^{-12} \mathrm{s.}^{2-4}$ Inserting $\tau_{m}=100 \mathrm{~s}$ and $\tau_{M}=5 \times 10^{-9} \mathrm{~s}$, we find that the ratio $T_{B}^{M} / T_{B}^{m}$ should be in the range $4-7$. Because of the particle size distribution, the peak temperature $T_{\max }$ in ZFC magnetization measurements may be larger than $T_{B}^{m}$ by a factor $\beta=1-2$. Therefore one would expect that the ratio $T_{B}^{M} / T_{\max }(0)$ is in the range $2-7$. For noninteracting maghemite particles with diameters of about $7.5 \mathrm{~nm}$, the ratio is of the order of $5 .^{11}$ For isolated particles one would also expect that the blocking temperatures, estimated from the ZFC magnetization curve and from measurements of the isothermal remanence, should be identical. The results of the present measurements on maghemite particles show a quite different behavior. The values of $T_{B}^{M}$ and $T_{\max }(0)$ are almost identical for the powder samples, but there is a large difference between the values of $T_{\max }$ and the blocking temperatures estimated from measurements of isothermal remanence. These results as well as the changes induced by coating and pressing of the particles clearly show that the magnetic interaction between the particles has a strong influence on the magnetic properties of the samples. In the powder samples, the separation between the particles is small, and it is therefore likely that strong magnetic interactions can result in the formation of an ordered state with a spin-glass-like structure (a super-spinglass). ${ }^{7,9,22,28,29}$ All the results of the present studies of maghemite particles are in fact consistent with such a model.

The almost identical values of $T_{\max }(0)$ and $T_{B}^{M}$ indicate that these temperatures are not superparamagnetic blocking temperatures, but the results are in accordance with a transition from a super-spin-glass state to a superparamagnetic state. ${ }^{29}$ It is well known that for spin glasses the value of $T_{\max }$ is close to the ordering temperature $^{40}$ and the magnetic hyperfine splitting in Mössbauer spectra is also expected to collapse at the transition temperature if the superparamagnetic relaxation is fast compared to the time scale of this technique. The observed variations of $T_{B}^{M}$ and $T_{\max }$ with the average interparticle distance thus reflect that the transition temperature increases when the strength of the interactions increases. The ratio $T_{B}^{M} / T_{\max }$ is about 1.4 for the ferrofluid, i.e., lower than expected for noninteracting particles. This indicates that even in the ferrofluid there is a significant influence of interactions. It is likely that the bigger particles in the ferrofluid form agglomerates which may exhibit ordering even above $100 \mathrm{~K}$.

The temperature dependence of the shape of Mössbauer spectra of small particles also gives information about interaction effects. For noninteracting particles with a particle size distribution, the spectra typically consist of a doublet due to small particles with a short relaxation time and a sextet due to larger particles with a longer relaxation time. ${ }^{41,42}$ The relative area of the doublet increases with increasing temperature because of the decreasing relaxation time. The splitting of the sextet is slightly smaller than that observed for bulk materials because of the influence of collective magnetic excitations, which leads to a reduction in the observed hyperfine field of up to $15 \% .^{25,42}$ In the case of strong interactions between nanoparticles, an ordered state can be found at low temperatures. The order parameter decreases continuously with increasing temperature, and the magnetic hyperfine field will vary proportionally to the order parameter provided that the relaxation is fast. $7,25,26,43,44$ Because of local variations in the strength of interactions, there may be a distribution of order parameters in a sample leading to a distribution of magnetic hyperfine fields in Mössbauer spectra. Therefore Mössbauer spectra of interacting particles typically exhibit much broader lines than spectra of noninteracting particles. ${ }^{7,9,43}$

When comparing the spectra shown in Figs. 2-4, it can be seen that the way in which the magnetic hyperfine field collapses is qualitatively different for the coated and uncoated samples. The spectra of the coated samples [Figs. 2, 3(a), and 4(a)] show the presence of both a quadrupole doublet and a six-line component in a broad temperature range. This indicates that some of the particles exhibit fast superparamagnetic relaxation and these particles thus interact only weakly with their neighboring particles. The lines of the sextets in the transition region are, however, broader than typically observed for noninteracting particles, and this suggests that the interaction between many of the particles is significant. The spectra of the uncoated particles, especially after pressing at 1280 MPa [Fig. 4(b)], show much more broadened lines and the quadrupole doublet is only visible for $T \gtrsim 234 \mathrm{~K}$. 
This behavior is consistent with the expected behavior of strongly interacting particles. The Mössbauer spectra therefore support the conclusion that the magnetic properties of the samples are strongly influenced by interactions and that the transition to the superparamagnetic state should not be explained by a simple change in the relaxation time with temperature [as described by Eq. (1)], but as a transition from a spin-glass-like state to a superparamagnetic state.

The blocking temperatures estimated from the isothermal remanence are much lower than those obtained from ZFC magnetization measurements, and the values of $M_{r}(0) / M_{s}(0)$ are about 0.35 . For noninteracting particles it is expected that the two blocking temperatures are identical and that $M_{r}(0) / M_{s}(0)=0.5$ for particles with uniaxial anisotropy. However, in systems of interacting particles, the extrapolated remanence is expected to be lower because of more or less complete flux closure. ${ }^{45}$ When the field is switched off at a low temperature, the sample will initially end up in a partly disordered state with a preferred orientation of the magnetic moments close to the applied field direction. It is unlikely that this is the state of lowest energy. The different spin-glass-like configurations are separated by energy barriers with heights that are determined by both the magnetic anisotropy and the magnetic interaction, and the system will decay to configurations of lower energy with a relaxation time which reflects the distribution in height of these barriers.

El-Hilo, O'Grady, and Chantrell ${ }^{5}$ found that the blocking temperatures, obtained from measurements of isothermal remanence for ferrofluids with different concentrations of 7.8-nm iron oxide particles, were nearly identical, and they concluded that the decay of remanence was not sensitive to interaction effects. Our results (Table II) show that there may be a slight increase in the barriers when going from the suspended to the coated dried and the uncoated samples, and that after pressing the uncoated particles at $1280 \mathrm{MPa}$ there is an increase in $T_{B}^{r}$ by about $50 \%$. These observations suggest that the energy barriers are mainly determined by the anisotropy and, except for the uncoated pressed sample, the distribution in barrier heights is only slightly changed by interactions. For the present particles, $K \approx 3.5 \times 10^{4} \mathrm{~J} \mathrm{~m}^{-3} .{ }^{31}$ This corresponds to $K V / k \approx 560 \mathrm{~K}$. If we take the values of $T_{B}^{M}$ (Table I) as a measure for the strength of the interaction, we find that $K V / k T_{B}^{M} \approx 4$ for the coated suspended sample, but the ratio decreases to about 2 for the uncoated pressed sample. These values indicate that the anisotropy may be predominant in the samples with the largest particle separation, but that the interaction becomes more important in the uncoated, pressed sample.

\section{CONCLUSIONS}

The influence of magnetic interactions between $7.5-\mathrm{nm}$ maghemite particles has been studied by use of Mössbauer spectroscopy and magnetization measurements. The apparent blocking temperatures obtained from Mössbauer spectroscopy $\left(T_{B}^{M}\right)$ and ZFC magnetization curves $\left(T_{B}^{m}\right)$ are almost identical when the particle separation is small, but the values obtained from isothermal remanence measurements $\left(T_{B}^{r}\right)$ are much lower. For noninteracting and weakly interacting particles, the characteristic temperatures are superparamagnetic blocking temperatures and the values of $T_{B}^{M}$ are then considerably larger than those of $T_{B}^{m}$, whereas the values of $T_{B}^{m}$ and $T_{B}^{r}$ should be identical. The present results show that the behavior of the samples is not governed only by a temperature-dependent superparamagnetic relaxation time. It is shown that all the results can be explained by the formation of a spin-glass-like ordered state at low temperatures. Thus the critical temperatures can be explained by a transition from a spin-glass-like ordered state to a superparamagnetic state, and the observed increase in the apparent blocking temperatures with decreasing interparticle separation is not due to an increasing superparamagnetic relaxation time, as has been suggested in earlier work on interacting particles, but it reflects an increase of the ordering temperature with increasing strength of interactions.

\section{ACKNOWLEDGMENTS}

The authors are grateful to K. O'Grady for the use of experimental facilities for magnetization measurements and to K. J. Davies for assistance with sample preparation. The authors also wish to thank M. El-Hilo for assistance with the magnetization measurements and S. W. Charles for valuable discussions. The work was supported by the EEC science program and the Danish Council for Technical Research.
*Present address: Materials Department, Risø National Laboratory, DK-4000 Roskilde, Denmark.

${ }^{1}$ L. Néel, Ann. Geophys. 5, 99 (1949).

${ }^{2}$ C. Johansson, M. Hanson, P. V. Hendriksen, and S. Mørup, J. Magn. Magn. Mater. 122, 125 (1993).

${ }^{3}$ S. Linderoth, L. Balcells, A. Laberta, J. Tejada, P. V. Hendriksen, and S. A. Sethi, J. Magn. Magn. Mater. 124, 269 (1993).

${ }^{4}$ D. P. E. Dickson, N. M. K. Reid, C. Hunt, H. D. Williams, M. El-Hilo, and K. O’Grady, J. Magn. Magn. Mater. 125, 345 (1993).

${ }^{5}$ M. El-Hilo, K. O’Grady, and R. W. Chantrell, J. Magn. Magn.
Mater. 114, 295 (1992).

${ }^{6}$ J. I. Gittleman, B. Abeles, and S. Bozoeski, Phys. Rev. B 9, 3891 (1974).

${ }^{7}$ S. Mørup, M. B. Madsen, J. Franck, J. Villadsen, and C. J. W. Koch, J. Magn. Magn. Mater. 40, 163 (1983).

${ }^{8}$ J. Jing, F. Zhao, X. Yang, and U. Gonser, Hyperfine Interact. 54, 571 (1990).

${ }^{9}$ I. Tamura and M. Hayashi, J. Magn. Magn. Mater. 72, 285 (1988).

${ }^{10} \mathrm{E}$. Tronc and J. P. Jolivet, in Studies of Magnetic Properties of Fine Particles and their Relevance to Material Science, edited 
by J. L. Dormann and D. Fiorani (Elsevier, New York, 1992), p. 199.

${ }^{11}$ P. Prené, E. Tronc, J. P. Jolivet, J. Livage, R. Cherkaoui, M. Noguès, J. L. Dormann, and D. Fiorani, IEEE Trans. Magn. MAG-29, 2658 (1993).

${ }^{12}$ S. Mørup and E. Tronc, Phys. Rev. Lett. 72, 3278 (1994).

${ }^{13}$ W. Luo, S. R. Nagel, T. F. Rosenbaum, and R. E. Rosensweig, Phys. Rev. Lett. 67, 2721 (1991).

${ }^{14}$ R. W. Chantrell, M. El-Hilo, and K. O'Grady, IEEE Trans. Magn. MAG-27, 3570 (1991).

${ }^{15}$ M. El-Hilo, K. O'Grady, and R. W. Chantrell, J. Magn. Magn. Mater. 114, 307 (1992).

${ }^{16}$ M. El-Hilo, K. O'Grady, and R. W. Chantrell, J. Magn. Magn. Mater. 117, 21 (1992).

${ }^{17}$ D. Fiorani, J. L. Dormann, J. L. Tholence, L. Bessais, and D. Villers, J. Magn. Magn. Mater. 54-57, 173 (1986).

${ }^{18}$ D. Fiorani, J. L. Tholence, and J. L. Dormann, J. Phys. C 19, 5495 (1986).

${ }^{19}$ J. L. Dormann, L. Bessasis, and D. Fiorani, J. Phys. C 21, 2015 (1988).

${ }^{20}$ J. L. Dormann, D. Fiorani, and M. El Yamani, Phys. Lett. A 120, 95 (1987).

${ }^{21}$ S. Shtrikman and E. P. Wohlfarth, Phys. Lett. 85A, 467 (1981).

22J. M. Luttinger and L. Tisza, Phys. Rev. 70, 954 (1946); 72, 257 (1947).

${ }^{23}$ J. P. Bouchard and P. G. Zérah, Phys. Rev. B 47, 9095 (1993).

${ }^{24}$ S. Romano, Phys. Rev. B 49, 12287 (1994), and references cited therein.

${ }^{25}$ S. Mørup, J. Magn. Magn. Mater. 37, 39 (1983).

${ }^{26}$ S. Mørup, P. H. Christensen, and B. S. Clausen, J. Magn. Magn. Mater. 68, 160 (1987).

${ }^{27}$ S. Mørup, IEEE Trans. Magn. MAG-23, 3518 (1987).

${ }^{28}$ M. A. Zaluska-Kotur and M. Cieplak, Europhys. Lett. 23, 85 (1993).
${ }^{29}$ S. Mørup, Europhys. Lett. 28, 671 (1994).

${ }^{30}$ P. V. Hendriksen, C. A. Oxborrow, S. Linderoth, S. Mørup, M. Hanson, C. Johansson, F. Bødker, K. Davies, S. W. Charles, and S. Wells, Nucl. Instrum. Methods Phys. Res. B 76, 138 (1993).

${ }^{31}$ S. Linderoth, P. V. Hendriksen, F. Bødker, S. Wells, K. Davies, S. W. Charles, and S. Mørup, J. Appl. Phys. 75, 6583 (1994).

${ }^{32}$ L. Gerward, S. Mørup, and H. Topsøe, J. Appl. Phys. 47, 822 (1976).

${ }^{33}$ R. W. Chantrell, J. Popplewell, and S. W. Charles, IEEE Trans. Magn. MAG-14, 975 (1978).

${ }^{34}$ P. H. Christensen, S. Mørup, and J. W. Niemantsverdriet, J. Phys. Chem. 89, 4898 (1985).

${ }^{35}$ Y. Maeda, M. Aramaki, Y. Takashima, M. Oogai, and T. Goto, Bull. Chem. Soc. Jpn. 60, 3241 (1987).

${ }^{36}$ S. Linderoth, M. D. Bentzon, and S. Mørup, Nucl. Instrum. Methods Phys. Res. B 76, 173 (1993).

${ }^{37}$ F. Bødker, S. Mørup, and S. Linderoth, Phys. Rev. Lett. 72, 282 (1994).

${ }^{38}$ T. Shinjo, T. Iwasaki, T. Shigematsu, and T. Takada, Jpn. J. Appl. Phys. 23, 283 (1984).

${ }^{39}$ A. Tari, R. W. Chantrell, S. W. Charles, and J. Popplewell, Physica 97B, 57 (1979).

${ }^{40}$ K. Binder and A. P. Young, Rev. Mod. Phys. 58, 801 (1986).

${ }^{41}$ W. Kündig, H. Bömmel, G. Constabaris, and R. H. Lindquist, Phys. Rev. 142, 327 (1966).

${ }^{42}$ S. Mørup, J. A. Dumesic, and H. Topsøe, in Applications of Mössbauer Spectroscopy, edited by R. L. Cohen (Academic, New York, 1980), Vol. II, p. 1.

${ }^{43}$ S. Mørup, Hyperfine Interact. 90, 171 (1994).

${ }^{44}$ S. Mørup, G. Christiansen, and N. C. Koon, J. Magn. Magn. Mater. 104-107, 1793 (1992).

${ }^{45}$ C. P. Bean and J. D. Livingston, J. Appl. Phys. 30, 1205 (1959). 